\title{
Intervenção nas situações de trabalho em um serviço de nutrição hospitalar e repercussões nos sintomas osteomusculares
}

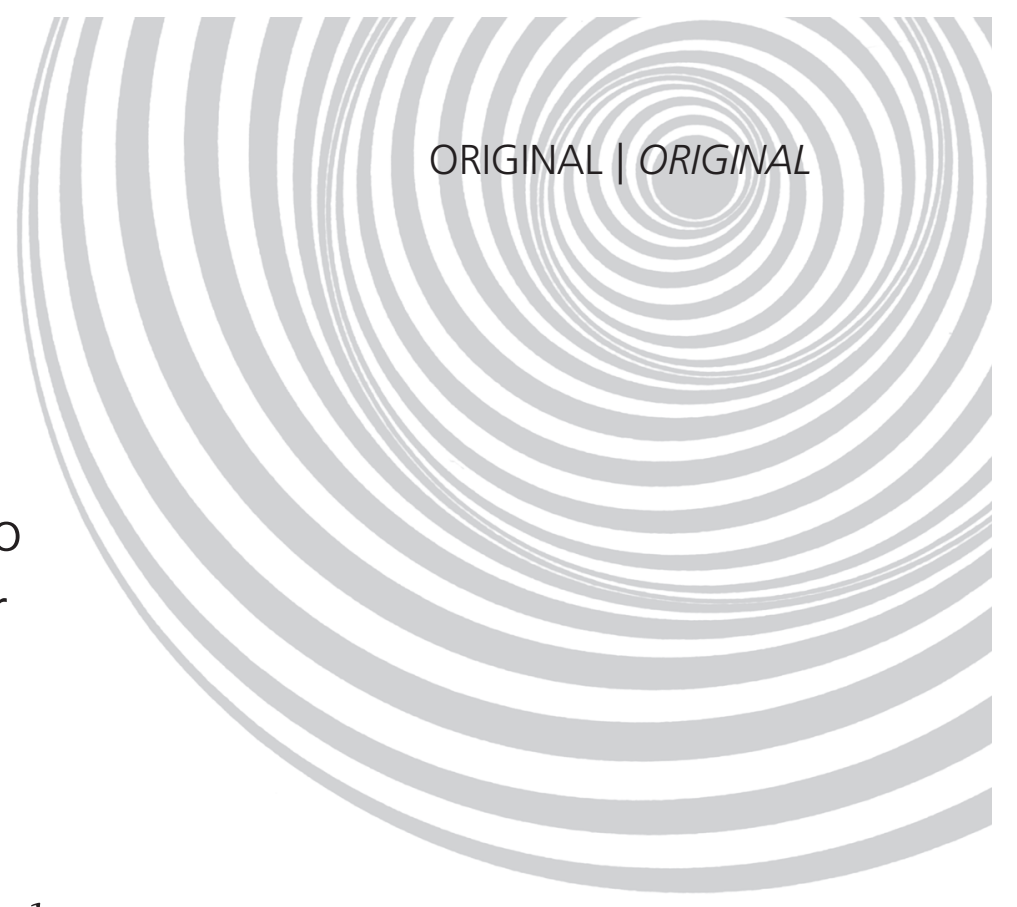

\author{
Intervention in a hospital foodservice and \\ its effects on musculoskeletal symptoms
}

\author{
Mitsue ISOSAKI ${ }^{1}$ \\ Elisabeth CARDOSO' \\ Débora Miriam Raab GLINA² \\ Marcelo PUSTIGLIONE ${ }^{3}$ \\ Lys Esther ROCHA4
}

\section{R E S U M O}

\section{Objetivo}

Analisar as situações de trabalho em serviço de nutrição hospitalar, antes e depois da implantação de ações de intervenções ergonômicas, e seu impacto na prevalência de sintomas osteomusculares relacionados ao trabalho.

\section{Métodos}

Trata-se de estudo de caso desenvolvido em hospital público especializado em cardiologia, localizado em São Paulo, com a participação de 115 trabalhadores. A abordagem metodológica foi a da Análise Ergonômica do Trabalho e da ergonomia participativa. A coleta de dados foi realizada por meio da aplicação de questionários e da análise ergonômica do trabalho, antes e depois da intervenção. A análise dos dados incluiu testes estatísticos para verificar se houve mudança da prevalência de sintomas antes e depois das intervenções, com nível de significância de 5\%, por meio dos Programas Statistical Package for Social Sciences 13.0 e Excel 2003.

\section{Resultados}

A população constitui-se, em sua maioria, de mulheres, na faixa etária de 25 a 34 anos, com grau médio de escolaridade, casadas, com filhos, e que ocupavam o cargo de atendente de nutrição. Os principais problemas

\footnotetext{
1 Universidade de São Paulo, Faculdade de Medicina, Instituto do Coração do Hospital das Clínicas, Serviço de Nutrição e Dietética. Av. Dr. Eneas de Carvalho Aguiar, 44, Cerqueira César, 05403-000, São Paulo, SP, Brasil. Correspondência para/Correspondence to: M. ISOSAKI. E-mail: <mitsue.isosaki@incor.usp.br>.

2 Universidade de São Paulo, Faculdade de Medicina, Hospital das Clínicas, Laboratório de Investigação Médica. São Paulo, SP, Brasil.

${ }^{3}$ Universidade de São Paulo, Faculdade de Medicina, Hospital das Clínicas, Serviço Especializado em Engenharia de Segurança e em Medicina do Trabalho. São Paulo, SP, Brasil.

${ }^{4}$ Universidade de São Paulo, Faculdade de Medicina, Departamento de Medicina Legal, Ética Médica e Medicina Social e do Trabalho. São Paulo, SP, Brasil.
} 
450 | M. ISOSAKI et al.

observados foram espaço físico reduzido, equipamentos e materiais de trabalho inadequados, deficit de pessoal, volume excessivo de trabalho com elevado esforço mental e alta prevalência de sintomas osteomusculares, principalmente nos membros inferiores e ombros. Após as intervenções realizadas, houve melhora na situação de trabalho, com redução dos sintomas osteomusculares, bem como os profissionais perceberam mudanças em termos de ambiente físico, equipamentos e organização do trabalho.

\section{Conclusão}

As intervenções repercutiram em melhorias, principalmente quanto ao ambiente e equipamentos, e na redução dos sintomas osteomusculares nos membros inferiores, ombros, pescoço/região cervical, antebraço e região lombar, apesar de a redução não ter sido estatisticamente significativa.

Termos de indexação: Condições de trabalho. Doenças profissionais. Saúde do trabalhador. Serviço hospitalar de nutrição. Trabalhadores.

\section{A B S T R A C T}

\section{Objective}

This study analyzed the working conditions of a hospital food service before and after ergonomic interventions and their impact on the prevalence of work-related musculoskeletal symptoms.

\section{Methods}

This case study was performed in a public heart hospital in São Paulo, Brazil with 115 workers. The data were collected by questionnaires and ergonomic analysis of the work before and after the intervention. The data were statistically analyzed by the software SPSS 13.0 and Excel 2003 to verify symptom prevalence before and after the interventions.

\section{Results}

The workers were female nutrition attendants aged 25-34 years, with secondary education, married, with children. Most of the problems regarded limited space, inappropriate equipment and work materials, inadequate number of workers, intense mental effort and high prevalence of musculoskeletal symptoms, mainly in the lower limbs and shoulders. Once the working conditions improved, musculoskeletal symptoms decreased and all workers noted the changes made to the physical environment, equipment and work organization.

\section{Conclusion}

The interventions promoted improvements in the work environment and equipment and reduced the musculoskeletal symptoms experienced in the lower limbs, shoulders, neck, forearm and lower back, although this reduction was not statistically significant.

Indexing terms: Working conditions. Occupational diseases. Occupational health. Food service. Workers.

\section{N T R O D U ÇÃ O}

O Serviço de Nutrição e Dietética (SND), inserido em hospitais brasileiros, tem por finalidade a prestação de assistência nutricional, por meio do fornecimento de refeições, orientação dietoterápica e educação alimentar. Em hospitais de ensino, desenvolve também programas de graduação e de pós-graduação, atividades de educação continuada, de pesquisa e de educação para a saúde da comunidade.

A característica principal desses serviços é a prestação diária, ininterrupta e contínua de aten- dimento aos pacientes. Suas atividades exigem exatidão, rapidez e sincronia da equipe. Os profissionais da área são submetidos a condições de trabalho adversas, como ruído, calor, umidade, risco de acidentes, esforço físico e/ou mental, ritmo de trabalho intenso, monótono e repetitivo, postura estática e sobrecarga muscular. Além disso, a saúde dos trabalhadores é afetada pela organização do trabalho, pelo estilo de liderança, pelo tipo de equipamentos e de utensílios adotados e pela forma como eles levantam, carregam e descarregam objetos $^{1-5}$.

O absenteísmo é um fator frequente, motivado geralmente por doenças, principalmente 
do sistema osteomuscular e do tecido conjuntivo ${ }^{5-7}$.

Estudos brasileiros realizados em cozinhas de restaurantes industriais e em serviços de nutrição hospitalares mostraram que os principais motivos das ausências foram as doenças do próprio trabalhador, sendo as do sistema osteomuscular as mais frequentes. Nesses estudos verificou-se ainda que fatores como ambiente de trabalho, espaço físico, equipamentos, organização e fatores psicossociais do trabalho geram repercussões na saúde dos trabalhadores ${ }^{3,5,6,8}$. O mesmo foi observado em outros países, em pesquisas realizadas em cozinhas de hotéis e restaurantes ${ }^{9-11}$.

Estudos de intervenção sobre sintomas osteomusculares, por meio da ergonomia participativa, têm sido realizados por pesquisadores do exterior ${ }^{12-16}$, porém no Brasil ainda são escassos, principalmente com trabalhadores de serviços de alimentação $23,17,18$.

A ergonomia participativa trata do envolvimento das pessoas no planejamento e controle de suas próprias atividades no trabalho, com conhecimento e poder suficientes para influenciar ambos os processos e resultados a fim de obter os objetivos desejados ${ }^{19}$.

Diante da escassez de avaliações de intervenções na área de nutrição hospitalar com base na ergonomia participativa, o objetivo do presente estudo foi analisar situações de trabalho em um serviço de nutrição hospitalar, antes e depois da implantação de ações de intervenção ergonômica, e seu impacto na prevalência de sintomas osteomusculares relacionados ao trabalho.

\section{M É T O D O S}

O estudo de caso, utilizando a metodologia da Análise Ergonômica do Trabalho (AET) de Guérin et al..$^{20}$ e ergonomia participativa ${ }^{12}$, foi realizado em um Serviço de Nutrição e Dietética de um hospital público, especializado em cardiologia, com 450 leitos, clínicos e cirúrgicos, localizado na cidade de São Paulo, Brasil. Do total de 129 trabalhadores, 115 participaram do projeto. As fases e duração da pesquisa são mostradas na Figura 1.

A demanda partiu de avaliações prévias da situação de trabalho ${ }^{18}$. Observou-se que dentre os principais motivos do absenteísmo destacavam-se as doenças do sistema osteomuscular e do tecido conjuntivo. Os trabalhadores relataram a necessidade de melhoria das condições de trabalho para diminuição do absenteísmo. Outro aspecto que demandou a análise da situação de trabalho foi o aumento do volume de refeições, decorrente da ampliação do número de leitos (de 32 para 450), sem ampliação proporcional do quadro de pessoal e da área física da cozinha. Esses fatos provavelmente refletiram na intensificação do trabalho e na maior exigência quanto ao desempenho dos trabalhadores.
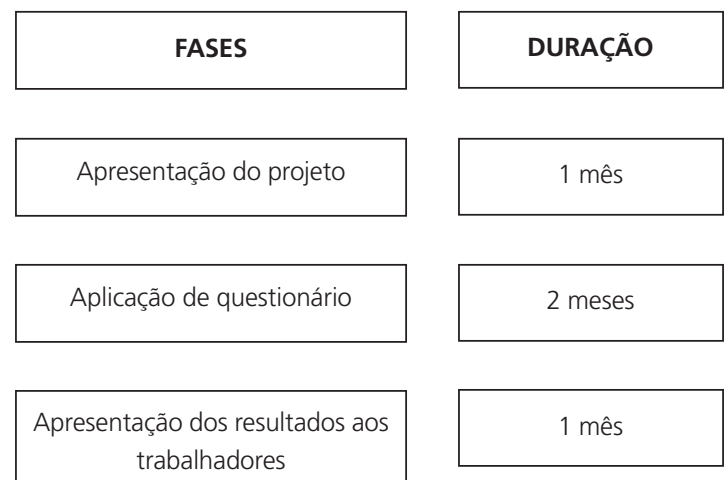

Apresentação dos resultados aos trabalho
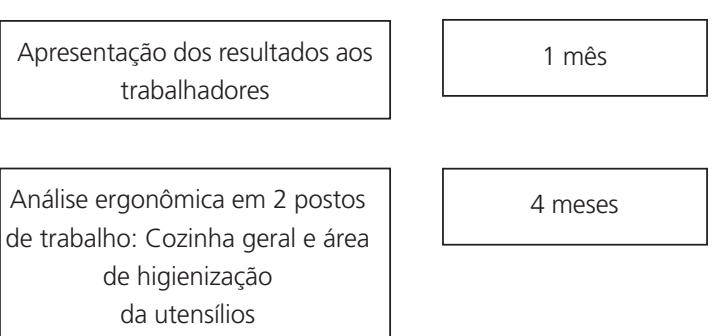

Implantação das intervenções

12 meses

Aplicação do questionário

2 meses

Reanálise ergonômica do trabalho

3 meses

Figura 1. Fases da pesquisa. São Paulo (SP), 2006-2007. 
O método de AET, conforme descrito por Guérin et al. ${ }^{20}$, permite um diagnóstico da situação de trabalho nos aspectos do ambiente, do posto, dos equipamentos, da organização e dos fatores psicossociais, visando transformar o trabalho para que os trabalhadores possam exercer suas competências sem alterar a saúde. A AET prevê 5 etapas: análise da demanda, que consiste na discussão e clarificação dos problemas a serem resolvidos; análise da tarefa, ou seja, tudo o que define o trabalho de cada um em uma dada estrutura; análise da atividade, isto é, dos comportamentos de trabalho; análise dos dados e autoconfrontação com os trabalhadores; diagnóstico e recomendações.

A demanda da AET incluiu três fases: apresentação do projeto aos trabalhadores, aplicação do questionário, coleta de sugestões de melhoria propostas pelos participantes.

Na primeira fase, o projeto foi apresentado aos trabalhadores, em grupos, nos horários de trabalho, a fim de esclarecer os objetivos e fases da pesquisa. A seguir, os trabalhadores foram convidados a participar do estudo, mediante termo de consentimento livre e esclarecido, após aprovação pela Comissão de Ética para Análise de Projetos de Pesquisa (CAPPesq), sob n 853/05.

Na segunda fase, foi aplicado um questionário aos 115 participantes, abordando dados sociodemográficos, história ocupacional, situações de trabalho e sintomas osteomusculares. As questões sobre sintomas osteomusculares foram baseadas no questionário nórdico de Kuorinka et al. ${ }^{21}$. As entrevistas foram individuais e duraram de 30 a 60 minutos, de acordo com o entendimento do trabalhador, tendo sido realizadas durante o horário de trabalho, por entrevistadores treinados e externos ao serviço.

Na terceira fase, foram apresentados os resultados dos questionários aos participantes, em reuniões em grupo, durante o horário de trabalho, ocasião em que eles apresentaram suas sugestões para melhoria das condições de trabalho.

Na quarta fase, a análise da tarefa, da atividade e o dignóstico foram realizados por espe- cialistas em ergonomia, externos à instituição. Foi selecionada a Seção de Produção de Alimentos (cozinha), por contar com $46 \%$ dos funcionários do serviço e por apresentar problemas críticos de espaço físico e leiaute. Dois postos de trabalho foram selecionados: cozinha geral e área de higienização de utensílios, cujas atividades demandavam maior sobrecarga muscular e movimentos repetitivos.

A análise da tarefa incluiu a avaliação dos riscos físicos e ambientais, realizada por um técnico em engenharia e segurança do trabalho, utilizando a metodologia da higiene ocupacional, em conformidade com a NR 15 do Ministério do Trabalho e NBR 5413 da Associação Brasileira de Normas Técnicas. Foram utilizados nas medições o medidor de nível de pressão sonora Simpson 886-2 Type Sound level meter para medição do ruído ambiental; o luxímetro digital Instrutherm LD-209, com fotocélula para captação do fluxo luminoso, para avaliação da luminosidade; e o medidor de temperatura e da umidade relativa do ar, com termômetro digital, Instrutherm modelo HT 100.

Para as medições dos pesos e das dimensões dos equipamentos e dos utensílios foram utilizadas balança digital Filizola, com capacidade de até 300 quilos; balança digital C\&F com capacidade mínima de $25 \mathrm{~g}$ e máxima de $3 \mathrm{~kg}$; e trena Stanley 3m/10', com graduação superior $1 / 16 \mathrm{e}$ inferior $0,0001 \mathrm{~m}$ tipo $1 \mathrm{~A}$.

A análise da atividade baseou-se em análises biomecânicas, realizadas por meio de entrevistas com os trabalhadores, filmagens ou registros fotográficos, e dinamometria com dinamômetro Lutron Force Gauge $110 \mathrm{~kg}$, Tension \& Compression.

Na quinta fase, foram implantadas ações relacionadas aos postos de trabalho, ambiente e aspectos organizacionais, baseadas nos resultados do questionário e nas recomendações dos ergonomistas, autoconfrontadas com os trabalhadores.

Na sexta fase, um segundo questionário, incluindo a avaliação da implementação, foi aplicado durante o horário de trabalho, mediante entrevista individual. Nessa fase, 89 trabalhadores 
responderam ao questionário. A ausência de 26 participantes foi devida a: demissão (13), aposentadoria (3), mudança para outro seviço (2), licença médica (2), licença-maternidade (1), férias (2) e recusa em participar (3).

Na sétima fase, foi realizada nova análise ergonômica, nos mesmos postos de trabalho, pelos mesmos especialistas e com uso das mesmas técnicas.

Para responder aos objetivos, foram feitas análises descritivas por meio de frequências absolutas e relativas das variáveis sociodemográficas, da história ocupacional, da situação do trabalho e da percepção dos trabalhadores. Para testes estatísticos para dados nominais, em amostras emparelhadas, foi utilizado o teste de McNemar ${ }^{22}$ para verificar se teria havido mudança estatisticamente significativa na opinião dos trabalhadores, antes e depois das intervenções realizadas, em relação à situação do trabalho e aos sintomas osteomusculares. Os programas utilizados para as análises foram o Statistical Package for Social Sciences (SPSS) 2003, Versão 13 para Windows, e o Excel 2003. O nível de significância adotado foi de $5 \%$.

\section{RES ULTADOS}

\section{Características}

A população constituiu-se, em sua maioria, de mulheres $(81 \%)$, casadas ( $53 \%)$, com grau de escolaridade médio (53\%) e média de idade de 37 (Desvio-Padrão - DP=9,8) anos. A maioria era atendente de nutrição (64\%), que trabalhava no hospital de 5 a 10 anos (22\%), em jornada de 40 horas semanais (78\%).

\section{Análise}

A análise ergonômica do trabalho descreve os resultados observados, explicitando o diagnóstico, apontando os principais condicionantes físico-ambientais e indicando as exigências físico-mentais do trabalho.
Nos dois postos de trabalho foi observada sobrecarga no sistema osteomuscular, principalmente sobre os membros superiores, devido à manipulação de equipamentos (caldeirão a vapor, forno combinado, batedeira) e utensílios (espátula para preparo de alimentos grelhados na chapa) e à higienização de louças. Durante essa fase, foi observado que um forno combinado e um caldeirão a vapor aguardavam manutenção, por problemas na porta e na tampa, respectivamente.

Na avaliação ambiental, embora o iluminamento estivesse dentro do limite recomendado pela legislação brasileira, havia lâmpadas sem proteção contra explosões e consideradas "cansadas e queimadas".

As principais exigências físicas do trabalho foram: desenvolver tarefas em pé; sustentar cargas acima da capacidade do trabalhador; realizar movimentos repetitivos e com uso dos mesmos grupos musculares; permanecer em posturas extremas; deslocar-se constantemente entre os postos de trabalho; expor-se ao frio, calor e ruído na execução das tarefas; realizar as atividades em ritmo acelerado, com apenas uma pausa oficial durante as jornadas de trabalho (8 e de 12 horas/dia).

Quanto às exigências cognitivas foi observada a necessidade de manter a qualidade do trabalho, realizar as tarefas sob pressão temporal, trabalhar com atenção concentrada e sustentar percepção visual para detalhes.

Em relação às exigências psíquicas, foram observadas as seguintes habilidades: saber trabalhar em equipe, ter controle sobre o próprio trabalho, adaptar-se às diferentes situações de trabalho, saber controlar o estresse imposto pelo volume e ritmo de trabalho, intercorrências do dia-a-dia e problemas nos relacionamentos interpessoais, bem como ter atitudes proativas e automotivação para desenvolver o trabalho, mesmo em situações desfavoráveis.

\section{Principais intervenções realizadas}

Foram implantadas modificações, envolvendo os trabalhadores e a equipe multidisciplinar, 
conforme mostrado no Quadro 1, abordando o ambiente físico, os equipamentos e utensílios, assim como a organização e os fatores psicossociais do trabalho.

\section{Percepção dos trabalhadores quanto às intervenções realizadas}

As opiniões dos trabalhadores sobre as condições de trabalho, antes e depois das inter- venções, estão apresentadas na Tabela 1. Em relação às condições ambientais, posto de trabalho, utensílios e organização do trabalho, houve um aumento percentual na percepção da melhoria nos itens iluminação, temperatura, qualidade dos utensílios, monotonia e repetitividade no trabalho e na liberdade para tomar decisões, embora essas diferenças não tenham sido estatisticamente significativas $(p>0,05)$. Quanto à avaliação dos itens "presença de passagens e corredores na cozinha"

Quadro 1. Modificações implantadas nas situações de trabalho estudadas e resultados obtidos. São Paulo (SP), 2006-2007.

\begin{tabular}{|c|c|c|}
\hline Condições físico-ambientais & Ação corretiva & Resultado \\
\hline Iluminação & Troca e reposicionamento das luminárias & $\begin{array}{l}\text { Elevação dos níveis de iluminamento das áreas: } \\
\text { - Cozinha geral: de } 478 \text { lux para } 621 \text { lux } \\
\text { - Higienização de utensílios: de } 219 \text { lux para } 480 \\
\text { lux }\end{array}$ \\
\hline Ruído & $\begin{array}{l}\text { - Cozinha Geral: substituição de válvula dani- } \\
\text { ficada do caldeirão a vapor } \\
\text { - Área de higienização de utensílios: troca dos } \\
\text { pratos de vidro por descartáveis }\end{array}$ & $\begin{array}{l}\text { Redução no nível de ruído das áreas: } \\
\text { - Cozinha geral: de } 92 \text { db para } 89 \text { db } \\
\text { - Higienização de utensílios: de } 92 \text { db para } 90 \\
\text { db }\end{array}$ \\
\hline Temperatura & $\begin{array}{l}\text { Acionamento do ar condicionado } 30 \text { minutos } \\
\text { antes do início da jornada de trabalho }\end{array}$ & $\begin{array}{l}\text { Melhoria no conforto térmico da cozinha, com } \\
\text { redução da temperatura de } 26,9^{\circ} \mathrm{C} \text { para } 24,3^{\circ} \mathrm{C}\end{array}$ \\
\hline \multicolumn{3}{|l|}{ Equipamentos e utensilios } \\
\hline Batedeira & $\begin{array}{l}\text { Compra de uma batedeira de menor capaci- } \\
\text { dade }(9 \mathrm{~kg}) \text { para preparo de alimentos em pe- } \\
\text { quenas quantidades }\end{array}$ & $\begin{array}{l}\text { Redução do esforço físico e da sobrecarga mus- } \\
\text { cular nos membros superiores }\end{array}$ \\
\hline Forno combinado & Conserto da porta que estava danificada & $\begin{array}{l}\text { Redução da força para abrir a porta de } 12 \mathrm{~kg} \text { para } \\
1 \mathrm{~kg} \text {, diminuindo o risco de sobrecarga nos mem- } \\
\text { bros superiores }\end{array}$ \\
\hline Caldeirão a vapor & $\begin{array}{l}\text { Conserto da tampa que estava com a mola } \\
\text { quebrada }\end{array}$ & $\begin{array}{l}\text { Redução da força para fechar o caldeirão de } 13 \mathrm{~kg} \\
\text { para } 4 \mathrm{~kg} \text {, diminuindo o risco de sobrecarga nos } \\
\text { membros superiores }\end{array}$ \\
\hline Carros para transporte de alimentos & Troca dos rodízios desgastados & $\begin{array}{l}\text { Redução de } 4 \mathrm{~kg} \text { na força necessária para em- } \\
\text { purrar/puxar os carros }\end{array}$ \\
\hline Espátula & $\begin{array}{l}\text { Aquisição de espátula de tamanho maior para } \\
\text { manusear alimentos na grelha, mais compatí- } \\
\text { vel com a quantidade preparada de alimentos }\end{array}$ & Eliminação de risco nos membros superiores \\
\hline Grelha removível em ferro fundido & $\begin{array}{l}\text { Substituição da grelha removível (que era usa- } \\
\text { da sobre o queimador do fogão) por equipa- } \\
\text { mento a gás fixo }\end{array}$ & $\begin{array}{l}\text { Eliminação do risco nos membros superiores, } \\
\text { uma vez que a grelha pesava } 17 \mathrm{~kg}\end{array}$ \\
\hline Pratos & $\begin{array}{l}\text { Substituição de pratos de vidro por descar- } \\
\text { táveis }\end{array}$ & $\begin{array}{l}\text { Redução do ciclo de trabalho para lavagem das } \\
\text { louças: de } 7-10 \text { segundos para } 2-3 \text { segundos, } \\
\text { impactando na redução de } 3 \text { horas para 1:45 } \\
\text { hora, para uma média de } 1060 \text { bandejas/dia, o } \\
\text { que representou uma diminuição importante no } \\
\text { movimento, no tempo e no ritmo de trabalho e } \\
\text { a eliminação de um posto de trabalho pela eli- } \\
\text { minação da operação "retirada de resíduos" }\end{array}$ \\
\hline
\end{tabular}


Quadro 1. Modificações implantadas nas situações de trabalho estudadas e resultados obtidos. São Paulo (SP), $2006-2007$.

\begin{tabular}{|c|c|c|}
\hline Condições físico-ambientais & Ação corretiva & Resultado \\
\hline Cesto de lixo & $\begin{array}{l}\text { Construção, pelo Serviço de Engenharia Hos- } \\
\text { pitalar, de suporte para elevação da altura do } \\
\text { cesto }\end{array}$ & $\begin{array}{l}\text { Diminuição da sobrecarga lombar para descar- } \\
\text { tar restos de alimentos }\end{array}$ \\
\hline $\begin{array}{l}\text { Equipamentos de proteção indivi- } \\
\text { dual }\end{array}$ & Aquisição de novos equipamentos & Introdução de oito novos equipamentos \\
\hline Esponja para lavagem de louças & $\begin{array}{l}\text { Substituição de esponja para lavagem de } \\
\text { louças por outra com espessura mais grossa }\end{array}$ & $\begin{array}{l}\text { Diminuição da sobrecarga no punho, mãos e de- } \\
\text { dos dos membros superiores }\end{array}$ \\
\hline \multicolumn{3}{|l|}{$\begin{array}{l}\text { Organização do trabalho fato- } \\
\text { res psicossociais }\end{array}$} \\
\hline Pausas & $\begin{array}{l}\text { Introdução, nos roteiros de atividades dos tra- } \\
\text { balhadores em jornada de trabalho de } 8 \text { ho- } \\
\text { ras/dia, de duas pausas de } 10 \text { minutos, em po- } \\
\text { sição sentada, após cada } 2 \text { horas de trabalho, } \\
\text { além das pausas oficiais }\end{array}$ & $\begin{array}{l}\text { Alternância de postura (em pé para sentada), } \\
\text { reduzindo a sobrecarga nos membros inferiores }\end{array}$ \\
\hline Grupo de reflexão & $\begin{array}{l}\text { Realizados } 12 \text { encontros com ergonomistas, } \\
\text { representantes dos trabalhadores e chefias, } \\
\text { com carga horária de } 42 \text { horas }\end{array}$ & $\begin{array}{l}\text { Estabelecimento de espaço coletivo para discus- } \\
\text { são, com melhorias no processo de trabalho e } \\
\text { na relação chefe/subordinado }\end{array}$ \\
\hline Treinamento para lideranças & $\begin{array}{l}\text { Desenvolvimento de um programa de treina- } \\
\text { mento para lideranças, com carga horária de } \\
30 \text { horas }\end{array}$ & $\begin{array}{l}\text { Melhoria da liderança situacional, do rapport e } \\
\text { feedback aos subordinados }\end{array}$ \\
\hline Ginástica laboral & $\begin{array}{l}\text { Realizada duas vezes na semana, por } 10 \text { mi- } \\
\text { nutos, durante } 4 \text { meses, e conduzida por es- } \\
\text { tagiária do curso de fisioterapia, sob supervi- } \\
\text { são }\end{array}$ & $\begin{array}{l}\text { Relaxamento, alongamento, prevenção e redu- } \\
\text { ção dos sintomas osteomusculares }\end{array}$ \\
\hline $\begin{array}{l}\text { Orientação postural para transpor- } \\
\text { te e manipulação de carga }\end{array}$ & $\begin{array}{l}\text { Orientação individual nos postos de trabalho, } \\
\text { durante } 4 \text { meses, conduzida por estagiárias do } \\
\text { curso de fisioterapia e de terapia ocupacional, } \\
\text { sob supervisão }\end{array}$ & $\begin{array}{l}\text { Adoção de posturas corretas, transporte de car- } \\
\text { gas com auxílio de carros ou em duplas }\end{array}$ \\
\hline
\end{tabular}

e "número de trabalhadores do serviço" foram considerados piores depois da intervenção $(p<0,05)$.

\section{Sintomas osteomusculares}

Dentre os 89 participantes, antes da intervenção 90\% deles referiram dor ou desconforto relacionado ao trabalho nos últimos 12 meses, contra $83 \%$ após a intervenção. Os membros inferiores foram as regiões mais afetadas (65\% na $1^{\text {a }}$ avaliação e $58 \%$ na $2^{a}$ ), seguidos pelo ombro ( $57 \%$ na $1^{\text {a }}$ avaliação e $47 \%$ na $2^{\text {a) }}$ (Tabela 2). A diferença percentual entre a $1^{\mathrm{a}}$ e a $2^{\mathrm{a}}$ avaliação não foi estatisticamente significativa $(p>0,05)$. Na Tabela 3 estão apresentadas as respostas dos trabalhadores quanto a causa, piora e melhora dos sintomas osteomusculares relacionados ao trabalho.

\section{I S C U S S Ã O}

Neste estudo verificaram-se condições de trabalho e arranjo físico inadequados, equipamentos por vezes insuficientes ou mal conservados, exigências físicas, cognitivas e psíquicas elevadas, dentre as quais se destacaram: volume excessivo de trabalho com ritmo acelerado sem pausa, longas horas de trabalho em pé; ciclos curtos; adoção de posturas extremas e antinaturais; uso acentuado de força; número excessivo de movimentos e deslocamentos constantes; trabalho monótono, 
456 | M. ISOSAKI et al.

Tabela 1. Opinião dos trabalhadores sobre condições de trabalho. São Paulo (SP), 2006-2007.

\begin{tabular}{|c|c|c|c|c|c|c|}
\hline \multirow{2}{*}{ Situação de trabalho } & & \multicolumn{2}{|c|}{ Antes da intervenção } & \multicolumn{2}{|c|}{ Após intervenção } & \multirow{2}{*}{$p$} \\
\hline & & $n$ & $\%$ & $\mathrm{n}$ & $\%$ & \\
\hline \multicolumn{7}{|l|}{ Ambiente } \\
\hline \multirow{3}{*}{ Ruído ( $n=89$ ) } & Excelente & 22 & 25 & 19 & 21 & \multirow{3}{*}{0,76} \\
\hline & Aceitável & 21 & 24 & 26 & 29 & \\
\hline & Inaceitável & 46 & 52 & 44 & 49 & \\
\hline \multirow[t]{3}{*}{ Iluminação ( $n=89$ ) } & Excelente & 59 & 66 & 68 & 76 & \multirow{3}{*}{0,16} \\
\hline & Aceitável & 20 & 22 & 15 & 17 & \\
\hline & Inaceitável & 10 & 11 & 6 & 7 & \\
\hline \multirow[t]{3}{*}{ Temperatura $(\mathrm{n}=89)$} & Excelente & 24 & 27 & 20 & 22 & \multirow{3}{*}{0,07} \\
\hline & Aceitável & 26 & 29 & 35 & 39 & \\
\hline & Inaceitável & 39 & 44 & 34 & 38 & \\
\hline \multicolumn{7}{|l|}{ Posto trabalho } \\
\hline \multirow[t]{3}{*}{ Espaço físico (n=89) } & Excelente & 18 & 20 & 21 & 24 & \multirow{3}{*}{0,51} \\
\hline & Aceitável & 24 & 27 & 24 & 27 & \\
\hline & Inaceitável & 47 & 53 & 44 & 49 & \\
\hline \multirow[t]{2}{*}{ Passagens e corredores $(n=89)$} & Sim & 12 & 13 & 2 & 2 & \multirow[b]{2}{*}{0,01} \\
\hline & Não & 77 & 87 & 87 & 98 & \\
\hline \multirow[t]{4}{*}{ Postura $(n=89)$} & Sentada & 11 & 12 & 16 & 18 & \multirow{4}{*}{0,33} \\
\hline & Em pé parado & 8 & 9 & 6 & 7 & \\
\hline & Em pé andando & 58 & 65 & 59 & 66 & \\
\hline & Variada & 12 & 13 & 8 & 9 & \\
\hline \multicolumn{7}{|l|}{ Utensílios } \\
\hline \multirow[t]{3}{*}{ Qualidade (n=89) } & Excelente & 25 & 28 & 25 & 28 & \multirow{3}{*}{0,73} \\
\hline & Aceitável & 29 & 33 & 35 & 39 & \\
\hline & Inaceitável & 35 & 39 & 29 & 33 & \\
\hline \multicolumn{7}{|l|}{ Organização e conteúdo do trabalho } \\
\hline \multirow[t]{2}{*}{ Monótono e repetitivo ( $n=89$ ) } & Sim & 55 & 62 & 51 & 57 & \multirow{2}{*}{0,52} \\
\hline & Não & 34 & 38 & 38 & 43 & \\
\hline \multirow[t]{2}{*}{ Esforço físico (n=89) } & Sim & 60 & 67 & 59 & 66 & \multirow{2}{*}{0,99} \\
\hline & Não & 29 & 33 & 30 & 34 & \\
\hline Esforço mental $(n=89)$ & Sim & 72 & 81 & 70 & 79 & 082 \\
\hline & Não & 17 & 19 & 19 & 21 & \\
\hline Volume excessivo ( $n=89$ ) & Sim & 65 & 73 & 70 & 79 & 0,38 \\
\hline & Não & 24 & 27 & 19 & 21 & \\
\hline Liberdade para tomar decisões $(n=89)$ & Sim & 26 & 29 & 30 & 34 & 0,54 \\
\hline & Não & 63 & 71 & 59 & 66 & 0,34 \\
\hline Número de trabalhadores $(n=83)$ & Suficiente & 24 & 29 & 12 & 14 & בחת \\
\hline & Insuficiente & 59 & 71 & 71 & 86 & 0,02 \\
\hline
\end{tabular}

Tabela 2. Prevalência de sintomas osteomusculares por região do corpo. São Paulo (SP), 2006-2007.

\begin{tabular}{|c|c|c|c|c|c|}
\hline \multirow{2}{*}{ Região do corpo } & \multicolumn{2}{|c|}{ Antes da intervenção } & \multicolumn{2}{|c|}{ Após intervenção } & \multirow{2}{*}{$p$} \\
\hline & $\mathrm{n}$ & $\%$ & $\mathrm{n}$ & $\%$ & \\
\hline Membros inferiores & 58 & 65 & 52 & 58 & 0,33 \\
\hline Ombros & 51 & 57 & 42 & 47 & 0,16 \\
\hline Lombar & 36 & 40 & 33 & 37 & 0,71 \\
\hline Pescoço/região cervical & 35 & 39 & 33 & 37 & 0,86 \\
\hline Região dorsal/coluna & 27 & 30 & 31 & 35 & 0,61 \\
\hline Punhos/mãos/dedos & 26 & 29 & 29 & 33 & 0,70 \\
\hline Antebraços & 25 & 28 & 22 & 25 & 0,70 \\
\hline Cotovelos & 9 & 10 & 9 & 10 & 0,99 \\
\hline
\end{tabular}


Tabela 3. Distribuição da opinião dos trabalhadores segundo causa, piora e melhora dos sintomas osteomusculares relacionados à atividade do trabalho. São Paulo (SP), 2006-2007.

\begin{tabular}{|c|c|c|c|c|}
\hline \multirow{2}{*}{ Sintoma } & \multicolumn{2}{|c|}{ Antes de intervenção } & \multicolumn{2}{|c|}{ Após a intervenção } \\
\hline & $n$ & $\%$ & $\mathrm{n}$ & $\%$ \\
\hline \multicolumn{5}{|l|}{ Causa } \\
\hline Deslocamento no trabalho & 47 & 32 & 42 & 21 \\
\hline Postura & 24 & 16 & 30 & 14 \\
\hline Levantamento/transporte/descarga material & 24 & 16 & 32 & 15 \\
\hline Organização do trabalho & 17 & 12 & 31 & 15 \\
\hline Condições ambientais & 13 & 9 & 15 & 7 \\
\hline Equipamentos/mobiliários & 9 & 6 & 21 & 10 \\
\hline Tensão emocional & 8 & 5 & 24 & 11 \\
\hline Outros & 6 & 4 & 14 & 7 \\
\hline \multicolumn{5}{|l|}{ Piora } \\
\hline Deslocamento no trabalho & 40 & 35 & 28 & 21 \\
\hline Tarefa & 28 & 25 & 23 & 17 \\
\hline Levantamento/transporte/descarga material & 17 & 15 & 21 & 15 \\
\hline Organização do trabalho & 13 & 11 & 21 & 15 \\
\hline Postura & 11 & 10 & 10 & 7 \\
\hline Tensão emocional & 1 & 1 & 15 & 11 \\
\hline Condições ambientais & 1 & 1 & 10 & 7 \\
\hline Outros & 2 & 2 & 10 & 7 \\
\hline \multicolumn{5}{|l|}{ Melhora } \\
\hline Tratamento com medicamento & 68 & 56 & 37 & 24 \\
\hline Repouso & 26 & 21 & 33 & 22 \\
\hline Alongamento & 13 & 10 & 31 & 20 \\
\hline Alteração de postura e pausa & 11 & 9 & 20 & 13 \\
\hline Melhoria no trabalho & 2 & 2 & 23 & 15 \\
\hline Nada & 2 & 2 & 9 & 6 \\
\hline
\end{tabular}

com grande pressão temporal; necessidade de atenção concentrada e memória; percepção e discriminação visual para detalhes; polivalência; flexibilidade; necessidade frequente de tomada de decisões; necessidade constante da colaboração dos chefes e colegas para a realização do trabalho, resultados semelhantes aos observados em cozinhas de unidades de alimentação e nutriçãó ${ }^{1-5,23-26}$.

A diferença principal entre a cozinha industrial e a hospitalar refere-se à exigência de atividade mental nesta última, devido à variabilidade no número e tipo de dietas e à responsabilidade na preparação de refeições destinadas ao tratamento dos pacientes, o que torna ainda mais crítico o trabalho desses profissionais.

Entre as repercussões na saúde dos profissionais, verificaram-se altas prevalências de sintomas osteomusculares, principalmente nos membros inferiores e no ombro, seguidas pelas regiões lombar e cervical. Estudos realizados no Brasil e no exterior com trabalhadores de cozinhas industriais e hotéis relatam também altas prevalências de sintomas nos membros inferiores ${ }^{5,24}$, nos ombros ${ }^{5,10,11}$ e na região lombar ${ }^{9-11}$.

As altas prevalências encontradas nos membros inferiores podem ser atribuídas à postura em pé, com deslocamentos e transporte de cargas, em jornadas de trabalho de 8 a 12 horas diárias. Segundo Bertoldi \& Proença ${ }^{26}$, o carregamento de peso, a postura, as condições ambientais, o volume de trabalho monótono e repetitivo e em ritmo intenso são fatores que contribuem para o desencadeamento ou agravamento de transtornos circulatórios nos membros inferiores.

Em relação ao ombro, os sintomas podem estar associados às posturas exigidas no abasteci- 
mento de alimentos em equipamentos de grande porte (caldeirões a vapor), no uso de utensílios de tamanho pequeno para misturar alimentos preparados em chapa, nas tarefas de manipulação de objetos pesados, assim como ao número excessivo de movimentos e/ou ao tempo prolongado na mesma postura. Pekkarinen \& Anttonen ${ }^{9}$ referem ainda o levantamento de peso acima da altura do ombro e a altura das superfícies de trabalho como causas.

Na região lombar, os sintomas podem ser explicados pela sobrecarga do sistema osteomuscular, pelo trabalho predominatemente em pé, pela postura exigida no carregamento de recipientes com alimentos sem o auxílio de carros de transporte, pelo peso dos mesmos, pela manutenção da postura de flexão de tronco e/ou número excessivo de movimentos de flexão. Pekkarinen \& Anttonen ${ }^{9}$ atribuem esses sintomas também ao trabalho em pé, em adição ao levantamento de peso a partir de baixas alturas.

Na região cervical, a prevalência observada pode estar relacionada às tarefas que exigem manutenção da flexão de pescoço e/ou número de movimentos excessivos de flexão, extensão, rotação e inclinação de pescoço.

No estudo de Jorge et al. ${ }^{8}$, realizado com a mesma população e com o objetivo de identificar a prevalência e os fatores de risco associados aos distúrbios osteomusculares relacionados ao trabalho, foram verificadas associações em relação ao ambiente (presença de ruído) e ao posto de trabalho (trabalho em pé a maior parte do tempo).

Nesta pesquisa foram realizadas intervenções usando a metodologia da ergonomia participativa e obteve-se redução dos sintomas osteomusculares em algumas regiões do corpo, porém não significativas estatisticamente. Isso pode ser justificado pela complexidade da situação de trabalho num serviço de nutrição e dietética, assim como pelas limitações das ações implantadas e pelo tempo de duração da avaliação.

Nos membros inferiores, a diminuição dos sintomas provavelmente decorreu da introdução de mais pausas oficiais e da possibilidade de estas serem realizadas no próprio posto de trabalho, em posição sentada.

Com relação aos sintomas nos ombros, a diminuição foi possivelmente decorrente da substituição e do conserto de equipamentos, da introdução de utensílios mais adequados, das orientações posturais nos postos de trabalho e da ginástica laboral. Essa redução pode ser considerada importante, uma vez que tal região do corpo é uma das que sofrem maior sobrecarga osteomuscular nas atividades desenvolvidas pelos trabalhadores, conforme observado nas análises biomecânicas realizadas, confirmando os achados de Pehkonen et al. ${ }^{27}$.

Quanto à região cervical houve pouca alteração, uma vez que ela é bastante solicitada nos inúmeros movimentos repetitivos observados nas análises biomecânicas da área de higienização de utensílios, com movimentos combinados de flexão, extensão e rotação.

As respostas indicando os fatores que contribuíram para a melhoria dos sintomas osteomusculares, após a realização da intervenção, mostraram redução da variável "medicamento", bem como aumento dos itens "melhorias no trabalho", "alongamento", "alteração da postura" e "realização de pausas". Esse resultado pode ser reflexo das orientações para o autocuidado, da introdução de pausas, da ginástica laboral e das orientações posturais.

O aumento da tensão emocional, tanto na causa como na piora dos sintomas osteomusculares, provavelmente está relacionado ao aumento da carga de trabalho devido à não substituição de funcionários aposentados.

Com relação aos fatores ambientais, as intervenções realizadas na iluminação e no nível de ruído propiciaram melhoria no conforto dos trabalhadores. Apesar da melhoria percebida no conforto térmico da cozinha, há de se ressaltar que a temperatura de $24,3^{\circ} \mathrm{C}$ ainda não atinge a recomendação da NR17 prevista no item 17.5.2, alínea $b$, que estabelece índice de temperatura entre $20^{\circ} \mathrm{C}$ e $23^{\circ} \mathrm{C}$. 
A compreensão dos resultados deste estudo está relacionada com o fato dos distúrbios osteomusculares relacionados ao trabalho serem de origem multicausal. Apesar de as intervenções realizadas envolverem ambiente, posto, equipamentos e organização do trabalho, não foram modificados aspectos fundamentais dessa organização. Da mesma forma, Haukka et al. ${ }^{14}$ também não encontraram diminuição significativa nos sintomas osteomusculares após uma série de intervenções ergonômicas. Para esses autores, parece que as mudanças não foram suficientemente intensas para influenciar os resultados na saúde ou o follow up precisaria ocorrer após um prazo maior, dada a natureza episódica dos distúrbios osteomusculares.

Os resultados podem refletir ainda a dificuldade de modificar as exigências físicas e mentais nas atividades do serviço de nutrição como um todo, o que necessitaria de um redesenho mais abrangente dos processos e da organização do trabalho. Segundo Dejours ${ }^{28}$, a ação ergonômica só pode ser considerada efetiva quando atinge a situação de trabalho em profundidade, principalmente a organização do trabalho. Neste estudo, embora esta tenha sido abordada, não foi possivel aumentar o número de trabalhadores para fazer frente às demandas crescentes de trabalho.

Estudos acerca dos impactos efetivos das intervenções sobre a saúde, por meio da ergonomia participativa, ainda são escassos na América Latina e também nos países mais desenvolvidos $^{12,15,17,29}$. Em estudos longitudinais, alguns resultados têm sido observados após 3 a 5 anos de acompanhamento ${ }^{16,30,31}$. Segundo Guérin ${ }^{20}$, a avaliação da intervenção ergonômica é complexa, pois esta pode ser bem-sucedida, mas inútil. Não existem critérios universais e também depende de quem julga. Além disso, uma contribuição ergonômica logo se mostrará limitada em função da evolução da empresa, de seu meio e das modificações das situações de trabalho. Conforme Haukka ${ }^{14}$ e Pater ${ }^{32}$, no mundo real, os problemas ergonômicos são sempre complexos, mas, quando bem compreendidos e resolvidos, geram ganhos em produtividade, redução do estresse e da fadiga, melhora das relações internas e estabilidade do quadro de pessoal capacitado.

De acordo com o relato dos técnicos que realizaram a análise ergonômica do trabalho e dos trabalhadores que participaram dos programas, houve impactos positivos na minimização dos fatores de risco, principalmente para distúrbios osteomusculares, observando-se ciclos de trabalho mais lentos, adoção de posturas mais confortáveis, execução de pausas entre as atividades, melhora na comunicação entre os pares e as chefias, maior envolvimento e participação dos trabalhadores nas resoluções dos problemas do serviço ${ }^{18}$.

O suporte dos gerentes e os treinamentos são muito importantes na eficácia das intervenções ergonômicas ${ }^{13,33}$. Atualmente, no Brasil, os hospitais vivem um dilema na área de recursos humanos, com a necessidade de modernização das formas de trabalho. Na busca da eficiência, o que tem sido comum é o corte de custos hospitalares por meio da dispensa de funcionários e da falta de investimento em treinamento profissional, causando impactos negativos à própria atividade do hospital, que é exclusivamente dependente de trabalho humano. Na instituição onde foi realizado o estudo, um aspecto importante foi a presença de um pedagogo, responsável pelo treinamento e desenvolvimento organizacional, que coordenava programas com foco na humanização e na motivação dos trabalhadores, de acordo com as necessidades de cada serviço e do trabalhador.

Por fim, pode-se acrescentar que, entre as limitações das ações implantadas que podem justificar a ausência de significância na redução dos sintomas, destaca-se a não ampliação do espaço físico da cozinha, desde sua inauguração há 30 anos, sem redimensionamento proporcional ao quadro de funcionários. Essa área, já na sua concepção e construção, apresentou deficiências tanto em relação ao espaço físico e equipamentos, quanto em relação ao fluxo de trabalho, uma vez 
que, na visão da maioria dos administradores, a área de alimentação não tem relação direta com a atividade-fim da empresa. Assim, para compensar as inadequações físicas, foram feitas adaptações nos procedimentos, fluxos e ritmos de produção, que potencializaram o impacto sobre a saúde dos trabalhadores, apesar das inovações introduzidas no serviço, como a aquisição de fornos combinados, bandejas térmicas e alimentos pré-processados, que trouxeram agilidade e simplificação nos processos de produção. De outro lado, a questão ergonômica não foi lembrada, provavelmente por falta de conhecimento dos próprios profissionais, além de não haver integração, desde o planejamento, entre administradores, arquitetos, engenheiros, profissionais da medicina do trabalho e usuários.

Outra questão é a pouca inovação tecnológica, além dos trâmites burocráticos, deficits orçamentários, baixos investimentos em saúde e baixo grau de escolaridade dos trabalhadores, dificultando a criação de ambiente favorável para melhor desempenho dos serviços de nutrição, dietética e alimentação, diferentemente do que ocorre em países mais desenvolvidos ${ }^{34,35}$. Como disseram Casarotto \& Mendes ${ }^{5}$, "a tecnologia deste século ainda não chegou para esses serviços, causando a seus trabalhadores sobrecarga, sofrimento e perda da saúde".

Neste estudo, apesar do cenário desfavorável, puderam ser observadas forças propulsoras e facilitadoras para a implantação de melhorias como a participação dos trabalhadores, o envolvimento das gerências do Serviço de Nutrição e Dietética, a colaboração dos profissionais do Serviço de Engenharia e Manutenção, do Serviço de Saúde e Segurança e de Gestão do Fator Humano da instituição, além do apoio dos especialistas em ergonomia externos à instituição.

A existência de poucos estudos, principalmente com trabalhadores de serviços de nutrição de hospitais, dificultou a comparação dos resultados. Como o presente trabalho é um estudo de caso, sugerem-se mais estudos de intervenção que avaliem as condições de trabalho e o impacto na saúde dos trabalhadores. Ainda assim, destaca-se a importância deste estudo multidisciplinar, baseado na análise ergonômica do trabalho e na ergonomia participativa, pois foi possível mostrar que, apesar dos recursos escassos, intervenções são possíveis e geram resultados. Nesse contexto, os profissionais responsáveis pelos serviços de nutrição hospitalar e saúde do trabalhador têm um papel fundamental a desempenhar, tanto na compreensão e na ampliação dos conhecimentos de ergonomia, quanto na identificação dos fatores de risco e na implantação de medidas preventivas exequíveis, para melhoria da situação de trabalho nesses importantes serviços em hospitais.

\section{O N CLUSÃO}

As intervenções resultaram em melhorias, principalmente quanto ao ambiente e equipamentos, e na redução dos sintomas osteomusculares nos membros inferiores, ombros, pescoço/região cervical, antebraço e região lombar, apesar de não significativa estatisticamente.

Para que ações de melhoria nas situações de trabalho, prevenção de doenças e promoção da saúde dos trabalhadores possam continuar sendo adotadas e avaliadas, recomenda-se a criação de um comitê permanente de ergonomia, envolvendo os trabalhadores, os profissionais de diferentes serviços da instituição e a direção da empresa.

\section{A GRADECIMENTOS}

À Fundação de Amparo à Pesquisa do Estado de São Paulo pelo apoio financeiro (processo $n^{\circ}$ 05/56 541-3), à equipe multidisciplinar e a todos os trabalhadores participantes da pesquisa.

\section{COLABORADORES}

M. ISOSAKI participou da concepção e do desenho do estudo; na obtenção de financiamento; na coleta, na análise e interpretação e análise estatística; na elaboração e na revisão crítica do manuscrito 
e supervisão do estudo. E. CARDOSO participou da coleta de dados; na elaboração e na revisão crítica do manuscrito. D.M.R. GLINA participou no apoio técnico das análises ergonômicas; na coleta de dados; na elaboração e na revisão crítica do manuscrito. M. PUSTIGLIONE participou na parte técnica e na revisão crítica do manuscrito. L.E. ROCHA concebeu o desenho do estudo; a obtenção do financiamento; analisou e interpretou os dados; elaborou e revisou o manuscrito.

\section{REFERÊ N CIAS}

1. Masculo FS, Souza DM, Seixas JWA, Moreira CRT. O serviço de nutrição e dietética hospitalar: um estudo de caso sob enfoque ergonômico. 1997. Disponível em: <www.abepro.org.br/biblioteca/ ENEGEP>.

2. Lima FPA, Araújo JNG, Souza RJ, Alves GBO. A produção das lesões por esforços repetitivos num restaurante universitário: análise ergonômica e psicossocial. In: Lima MEA, Araújo JNG, Lima FPA, Ferreira Junior M. LER: lesões por esforços repetitivos: dimensões ergonômicas e psicossociais. Saúde. 1998; 108-177.

3. Lancman S, Siqueira AR, Queiroz MFF, Varela RCB. Estudo e intervenção no processo de trabalho em um restaurante universitário: em busca de novas metodologias. Rev Ter Ocup Univ São Paulo. 2000; 11(2/3):79-89.

4. Matos $\mathrm{CH}$, Proença RPC. Condições de trabalho e estado nutricional de operadores do setor de alimentação coletiva: um estudo de caso. Rev Nutr. 2003; 16(4):493-502. doi: 10.1590/S1415-52732 003000400012 .

5. Casarotto RA, Mendes LF. Queixas, doenças ocupacionais e acidentes de trabalho em trabalhadores de cozinhas industriais. Rev Bras Saúde Ocup. 2003; 28(107/108):109-26.

6. Isosaki M. Absenteísmo entre trabalhadores de serviços de nutrição e dietética de dois hospitais em São Paulo-SP. Rev Bras Saúde Ocup. 2003; 28 (107/108):107-18.

7. Paiva AC, Cruz AAF. Estado nutricional e aspectos ergonômicos de trabalhadores de unidade de Alimentação e Nutrição. Rev Mineira Ciênc Saúde. 2009; 1(1):1-11.

8. Jorge AT, Glina DMR, Isosaki M, Ribeiro AC, Ferreira Júnior M, Rocha LE. Distúrbios osteomusculares do trabalho: fatores de risco em trabalhadores de nutrição hospitalar. Rev Bras Med Trab. 2009; 7:1-10.
9. Pekkarinen A, Anttonen $\mathrm{H}$. The effect of working height on the loading of the muscular and skeletal systems in the kitchens of workplace canteens. Appl Ergon. 1988; 194:306-8.

10. Haukka E, Leino-Arjas P, Solovieva S, Ranta R, Viikari-Juntura E, Riihimäki $\mathrm{H}$. Co-occurrence of musculoskeletal pain among female kitchen workers. Int Arch Occup Environ Health. 2006; 80(2): 141-8.

11. Chyuan JY, Du CL, Yeh WY, Li CY. Musculoskeletal disorders in hotel restaurant workers. Occup Med. 2004; 54(1):55-7.

12. Rivilis I, Eerd DV, Cullen K, Cole DC, Irvin E, Tyson $J$, et al. Effectiveness of participatory ergonomic intervention on health outcomes: a systematic review. App Ergon. 2008; 39(3):342-58.

13. Pehkonen I, Takala EP, Ketola R, Viikari-Juntura E, Leino-Arjas P, Hopsu L, et al. Evaluation of a participatory ergonomic intervention process in kitchen work. Appl Ergon. 2008; 40(1):115-23. doi:10.1016/j.apergo.2008.01.006.

14. Haukka E, Leino-Arjas P, Viikari-Juntura E, Takala EP, Malmivaara A, Hopsu L, et al. A randomized controlled trial on whether a participatory ergonomics intervention could prevent musculoskeletal disorders. Occup Environ Med. 2008; 65(12): 849-56.

15. Denis D, St-Vincent M, Imbeau D, Jetté C, Nastasia I. Intervention practices in musculoskeletal disorder prevention: a critical literature review. Appl Ergon. 2008; 39(1):1-14.

16. Devereux J. The long term impact of two participatory ergonomics programmes for reducing musculoskeletal injuries and improving work performance. In: Sznelwar L, Mascia F, Monteiro U, editors. Human factors in organizational design and managementIX. California: IEA Press; 2008. p.211-6.

17. Coury HJCG. Time trends in ergonomic intervention research for improved musculoskeletal health and comfort in Latin America. Appl Ergon. 2005; 36: 249-52.

18. Isosaki M. Intervenção nas situações de trabalho em um serviço de nutrição hospitalar de São Paulo e repercussões nos sintomas osteomusculares [tese]. São Paulo: Universidade de São Paulo; 2008. Disponível em: <http://www.teses.usp.br/teses/ disponiveis/5/5160/tde-13012009-115732/>.

19. Wilson JR, Haines H. Participatory ergonomics. In: Salvendy $G$, editor. Handbook of human factors and ergonomics. $2^{\text {nd }}$ ed. New York: Wiley; 1997. p.490-513.

20. Guérin F, Laville A, Daniellou F, Duraffourg J, Kerguelen A. Compreender o trabalho para 
462 M. ISOSAKI et al.

transformá-lo: a prática da ergonomia. São Paulo: Edgard Blücher; 2001.

21. Kuorinka I, Jonsson B, Kilbom A, Vinterberg $H$, Biering-Sorensen F, Andersson G. Standardised Nordic questionnaire for the analysis of musculoskeletal symptoms. Appl Ergon. 1987; 18(3):233-7.

22. Agresti A. Categorical data analysis. New York: Weley; 1990

23. Sant'ana HMP, Azeredo RMC, Castro JR. Estudo ergonômico em serviços de alimentação. Saúde Debate. 1994; 42:45-8.

24. Alves GBO. Contribuições da ergonomia ao estudo da LER em trabalhadores de um restaurante universitário [dissertação]. Santa Catarina: Universidade Federal de Santa Catarina; 1995.

25. Colares LGT, Freitas CM. Processo de trabalho e saúde de trabalhadores de uma unidade de alimentação e nutrição: entre a prescrição e o real do trabalho. Cad Saúde Pública. 2007; 23(12): 3011-20.

26. Bertoldi CML, Proença RPC. Doença venosa e sua relação com as condições de trabalho no setor de produção de refeições. Rev Nutr. 2008; 21(4):447-54. doi: 101590/S1415-52732008000400009.

27. Pehkonen I, Miranda H, Haukka E, Luukkonen R, Takala EP, Ketola $R$, et al. Prospective study on shoulder symptoms among kitchen workers in relation to self-perceived and observed work load. Occup Environ Med. 2009; 66(6):416-23.

28. Dejours C. A loucura do trabalho: estudo da psicopatologia do trabalho. São Paulo: Oboré; 1988.
29. Haukka E, Pehkonen I, Leino-Arjas P, Viikari-Juntura E, Takala EP, Malmivaara A, et al. Effect of a participatory ergonomics intervention on psychosocial factors at work in a randomized controlled trial. Occup Environ Med. 2010; 67:170-7.

30. Evanoff BA, Bohr PC, Wolf LD. Effects of a participatory ergonomics team among hospital ordelies. Am J Ind Med. 1999; 35(4):358-65.

31. Hignett S, Wilson JR, Morris W. Finding ergonomic solutions-participatory approaches. Occup Med. 2005; 55(3):200-7.

32. Pater R. A manager's ergonomic responsibility. Occup Health Saf. 1997; 66:87-94.

33. Morken T, Moen B, Riise T, Hauge SHV, Holien S, Langedrag $A$, et al. Effects of a training program to improve musculosketelal health among industrial workers: effects of supervisors role in the intervention. Int J Ind Ergonomics. 2002; 30:115-27.

34. Proença RPC. Novas tecnologias para a produção de refeições coletivas: recomendações de introdução para a realidade brasileira. Rev Nutr. 1999; 12(1):43-53. doi: 10.1590/S1415-527319990001 00004.

35. Sousa AA, Proença RPC. Tecnologias de gestão de cuidados nutricionais: recomendações para qualificação do atendimento nas unidades de alimentação e nutrição hospitalares. Rev Nutr. 2004; 17(4): 425-36. doi: 10.1590/S1415-52732004000400 003.

Recebido em: 28/8/2009

Versão final reapresentada em: 9/2/2011 Aprovado em: 15/3/2011 